\title{
Review Article \\ Cytokines in Bipolar Disorder: Paving the Way for Neuroprogression
}

\author{
Izabela Guimarães Barbosa, ${ }^{1}$ Moisés Evandro Bauer, ${ }^{2}$ \\ Rodrigo Machado-Vieira, ${ }^{3,4}$ and Antonio Lucio Teixeira ${ }^{1,5}$ \\ ${ }^{1}$ Divisão de Neuropsiquiatria, Laboratório Interdisciplinar de Investigação Médica, \\ Faculdade de Medicina, Universidade Federal de Minas Gerais (UFMG), Avenida Alfredo Balena, \\ 190 Santa Efigênia, 30130-100 Belo Horizonte, MG, Brazil \\ ${ }^{2}$ Laboratório de Imunologia do Envelhecimento, Instituto de Pesquisas Biomédicas, \\ Pontifícia Universidade Católica do Rio Grande do Sul (PUC-RS), 90610-000 Porto Alegre, RS, Brazil \\ ${ }^{3}$ Laboratório de Neurociências, LIM27, Instituto e Departamento de Psiquiatria, Universidade de Sao Paulo, \\ 01060-970 Sao Paulo, SP, Brazil \\ ${ }^{4}$ Experimental Therapeutics and Pathophysiology Branch, National Institute of Mental Health, \\ National Institutes of Health, Bethesda, MD 20852, USA \\ ${ }^{5}$ Instituto de Estudos Avançados Transdisciplinares (IEAT), UFMG, 31270-901 Belo Horizonte, MG, Brazil
}

Correspondence should be addressed to Izabela Guimarães Barbosa; izabelagb@gmail.com and Antonio Lucio Teixeira; altexr@gmail.com

Received 18 July 2014; Accepted 23 August 2014; Published 9 September 2014

Academic Editor: João Quevedo

Copyright (C) 2014 Izabela Guimarães Barbosa et al. This is an open access article distributed under the Creative Commons Attribution License, which permits unrestricted use, distribution, and reproduction in any medium, provided the original work is properly cited.

Bipolar disorder (BD) is a severe, chronic, and recurrent psychiatric illness. It has been associated with high prevalence of medical comorbidities and cognitive impairment. Its neurobiology is not completely understood, but recent evidence has shown a wide range of immune changes. Cytokines are proteins involved in the regulation and the orchestration of the immune response. We performed a review on the involvement of cytokines in $\mathrm{BD}$. We also discuss the cytokines involvement in the neuroprogression of $\mathrm{BD}$. It has been demonstrated that increased expression of cytokines in the central nervous system in postmortem studies is in line with the elevated circulating levels of proinflammatory cytokines in $\mathrm{BD}$ patients. The proinflammatory profile and the immune imbalance in $\mathrm{BD}$ might be regarded as potential targets to the development of new therapeutic strategies.

\section{Introduction}

Bipolar disorder (BD) is a chronic, severe, and disabling medical illness. The lifetime prevalence is estimated at $2.4 \%$ [1]. The cardinal diagnostic feature is the occurrence of at least one episode of mania and/or hypomania, although depressive episodes tend to predominate in the course of the illness. The mean age at onset is around 20 years old. The risk of mood episodes remains constant after 40 years of the illness, indicating a risk of recurrence of symptoms until around 70 years of age [2]. BD patients present significant mood symptoms during at least half of their lifetime [3]. The cyclic nature of manic and depressive symptoms has been appointed as the major cause of disability in BD patients [4].

$\mathrm{BD}$ is characterized by a temporal progression of symptoms, that is, increase in the frequency and severity of mood episodes and less response to treatment [5-8]. Several studies have also reported cognitive impairment along with structural (decrease of hippocampal and amygdala volumes and total gray matter) and neurophysiological changes [59]. The term "neuroprogression" was applied to refer to this temporal clinical progression in $\mathrm{BD}$ based on the notion of clinical staging used in oncology and internal medicine. One of the main factors associated with the neuroprogression 
and, consequently, with the prognosis is the frequency of mood episodes (mania or depression). Hence the higher the frequency of mood episodes is associated with the fastest neuroprogression changes and the compromised prognosis. The biological mechanisms underlying BD neuroprogression are not determined and may involve complex interactions among multiple genes and environmental factors leading to impairment in several physiological systems [5].

Indeed $\mathrm{BD}$ has been regarded as a multisystemic condition, impairing cognitive, endocrine, autonomic, and circadian rhythms. There is an elevated incidence of psychiatric disorders like anxiety disorders, obsessive compulsive disorder, alcohol and substance abuse, attentiondeficit/hyperactive disorder, and eating disorders [1, 10]. $\mathrm{BD}$ is frequently comorbid with several medical conditions, including cardiovascular and metabolic diseases (particularly diabetes mellitus and obesity) that partially contribute to the reduced lifetime expectancy in these patients [11, 12]. The cooccurrence of autoimmune diseases has also been described. For instance, case-control studies showed that BD patients present high frequency of systemic lupus erythematosus [13], multiple sclerosis [14, 15], and autoimmune thyroiditis [16]. Recently a cohort study showed that a history of Guillain-Barre syndrome, Crohn's disease, or autoimmune hepatitis was associated with a raised risk of BD [17].

A growing body of evidence, represented mainly by the finding of increased circulating levels of proinflammatory cytokines, suggests that immune-mediated mechanisms are related to the neurobiology of $\mathrm{BD}$ and its neuroprogression. Cytokines, a broad category of small proteins, are traditionally involved in the orchestration of immune responses [18]. Besides this classical role, they can directly affect neuronal activity, inducing neuronal excitability and plastic changes [19]. Moreover cytokines can influence the hypothalamicpituitary-axis (HPA) through effects on the HPA feedback regulation and on the glucocorticoid receptor function [20]. Cytokines activate the HPA axis, increasing the levels of corticotrophin releasing hormone $(\mathrm{CRH})$, adrenocorticotropic hormone (ACTH), and cortisol and decreasing the expression, translocation, and downstream effects of glucocorticoid receptors [21]. The resulting net effect is a persistent elevation of glucocorticoids levels which has been consistently associated with mood symptoms [22]. Cytokines may also interfere with the metabolism of neurotransmitters, such as serotonin and dopamine, in determined brain regions (amygdala, hippocampus, and nucleus accumbens) involved in the regulation of emotion, reward, and psychomotor functions $[23,24]$. Indeed both proinflammatory cytokines can stimulate the enzyme indoleamine 2,3-dioxygenase (IDO) [25]. IDO converts tryptophan into kynurenine (KYN), reducing the availability of this precursor of serotonin [2226]. Proinflammatory cytokines also enhance the activity of the enzyme kynurenine-3-monooxygenase enzyme (KMO) that degrades KYN into 3-hydroxykynurenine, shifting the KYN pathway into the production of neurotoxic metabolites.

In the present paper, we performed a comprehensive review on the role of cytokines in $\mathrm{BD}$, also addressing their involvement in illness progression. The following search strategy was applied: online search of the databases
MEDLINE and SCOPUS from 1990 until May 2014 using the keywords (MESH criteria): "bipolar disorder" AND "cytokines." Only papers published in English were evaluated.

\section{Evidence of Altered Cytokines in Bipolar Disorder}

2.1. Evidence of Cytokine-Related Genes in Bipolar Disorder. $\mathrm{BD}$ is strongly influenced by genetic factors. The concordance in monozygotic twins is $67 \%$ and the concordance in dizygotic twins is $19 \%$, and the estimate heritability is around 80\% [27]. Among several candidate genes, some studies investigated the association between $\mathrm{BD}$ and immune-related genes polymorphisms.

Two case-control studies reported that $\mathrm{BD}$ is associated with an uncommon variant with an adenine (A) at position -308 of the tumor necrosis factor alpha (TNF- $\alpha$ ) gene which is related with higher TNF- $\alpha$ production $[28,29]$. However, two other case-control studies failed to confirm such association [30,31].

Regarding the interleukin- (IL-) 1 family, four casecontrol studies evaluated its expression and polymorphisms in $\mathrm{BD}$. In $\mathrm{BD}$ patients, the expression of a genetic variant of the IL- $1 \beta$ gene, the variant $(-511 \mathrm{~T})$, was associated with volume decrease of the gray matter, especially in the left dorsolateral prefrontal cortex, indicating a role for proinflammatory mechanisms in brain structural changes [32]. Two studies demonstrated that the presence of a variable number of tandem repeats (VNTR) in intron 2 of the IL-1Ra gene (IL1RN) (IL1RN*2) confers susceptibility to BD [33], particularly in $\mathrm{BD}$ patients with a positive family history of the illness [34]. Nevertheless, a third study failed to confirm this finding [35]. Interestingly, IL1RN ${ }^{*} 2$ allele was associated with more prolonged and more severe proinflammatory immune response when compared with other IL1RN genotypes in humans. In vitro studies also demonstrated that the presence of the IL1RN* 2 allele was associated with enhanced IL-1 $\beta$ production and decreased production of IL-1Ra $[36,37]$.

Three case-control studies revealed that $\mathrm{BD}$ patients present a significant association between the uncommon variant with an adenine (A) at position -2581 of the CCL2 gene that is associated with higher production of the chemokine CCL2/MCP-1 (involved in the recruitment of monocytes, memory $\mathrm{T}$ cells and dendritic cells into inflammatory sites) under proinflammatory stimulus [3840]. Despite the reported associations between BD and immune-related genes, none of them has been confirmed by meta-analyses including genome-wide association studies $[41,42]$.

Regarding transcriptomic changes, Padmos et al. (2008) [43] reported that monocytes from BD patients and their offspring had overexpression of mRNA cytokines, notably TNF$\alpha$, IL- $1 \beta$, IL- 6 , and CCL2. One hypothesis is that the aberrant RNA processing of cytokines in $\mathrm{BD}$ might be determined by (not yet defined) epigenetic mechanisms influencing not only cytokines levels but also the neurobiology of the illness. 
2.2. Neuroimaging and Postmortem Studies and Their Relationship with Cytokines in Bipolar Disorder. There are still a limited number of studies evaluating brain abnormalities and cytokines in $\mathrm{BD}$ patients at the same time point. Hsu et al. [44] found that serum levels of IL-10 correlated with serotonin transporter availability (as assessed by single-photon emission computed tomography (SPECT)) in the thalamus, indicating a possible link between peripheral inflammation and monoamine metabolism in the brain. Conversely, one study failed to find any association between peripheral IL-1 receptor antagonist (IL-ra) levels and white matter integrity [45].

Postmortem studies demonstrated the presence of dendritic atrophy of neurons and the loss of oligodendroglial cells in $\mathrm{BD}$, notably in the medial prefrontal cortex [46]. Only few studies have assessed immune markers in this context, revealing increased inflammatory and decreased anti-inflammatory markers in the frontal cortex of BD patients compared with controls $[47,48]$. More specifically, they reported increased protein and mRNA levels of IL-1 $\beta$ and its receptor (IL-1R), NF- $\kappa$ B transcription factor subunits (p50 and p65), and astroglial and microglial markers (glial fibrillary acidic protein, inducible nitric oxide synthase, $c$-fos and CD11b) [47]. Conversely, BD patients showed decreased total RNA expression of transforming growth factor (TGF) $\beta$ in the frontal cortex [47]. Dean et al. [49] also showed that BD patients present increased transmembrane TNF- $\alpha$ protein level in the anterior cingulate area and decreased TNF- $\alpha$ receptor 2 protein levels in the dorsolateral prefrontal cortex in comparison with controls. Since the dorsolateral prefrontal cortex is known to be critical in executive functioning, the anterior cingulate in mood control and all these regions are implicated in $\mathrm{BD}$ [50]; these data suggest a role for cytokines in disease pathogenesis and also in mood and cognition modulation. Nevertheless, studies assessing whether there is any association between neuropathological findings and clinical (cognitive or psychopathological) parameters are lacking.

Taking into account that microglia is one of the major sources of cytokines in the central nervous system (CNS), these cells were investigated in BD. Postmortem studies reported decreased number and size of microglia in $\mathrm{BD}$ patients [51]. To conciliate these contradictory results, it was hypothesized that persistent microglia activation in early stages is associated with cellular death in the long term due to failure in the control of anabolic and catabolic cellular machineries, corroborating the view of a neurodegenerative process in $\mathrm{BD}$ [52].

2.3. Circulating Cytokine Levels in Bipolar Disorder. Four meta-analyses evaluated peripheral levels of cytokines in BD patients in comparison with controls [53-56]. The concentrations of IL-4, TNF- $\alpha$, sTNFR1, and sIL-2R were significantly higher in BD patients in comparison with controls [53-56]. There were no significant differences between $\mathrm{BD}$ patients and controls for IL-1 $\beta$, IL-2, IL-5, IL-6, IL-8, IL-12, IFN$\gamma$, TGF- $\beta 1$, IL-1ra, and sTNFR2 [53-56]. A meta-analysis assessing specifically chemokines did not find any association with BD [56].
TABLE 1: Cytokine peripheral levels in bipolar disorder patients in euthymia compared with controls.

\begin{tabular}{|c|c|c|}
\hline Cytokine & Findings & Conclusion \\
\hline \multirow{2}{*}{ TNF } & $\leftrightarrow[44,57,61,62,73,77,85,86,101-105]$ & \multirow{2}{*}{$\leftrightarrow$} \\
\hline & $\uparrow[63,70]$ & \\
\hline \multirow{2}{*}{ sTNFR1 } & $\leftrightarrow[73,106,107]$ & \multirow{2}{*}{$\uparrow$} \\
\hline & $\uparrow[61,103,105,108]$ & \\
\hline \multirow{2}{*}{ sTNFR2 } & $\leftrightarrow[61,73,103]$ & \multirow{2}{*}{$\leftrightarrow$} \\
\hline & $\uparrow[105]$ & \\
\hline \multirow{2}{*}{ IFN- $\gamma$} & $\leftrightarrow[63,86]$ & \multirow{2}{*}{$\mathrm{NC}$} \\
\hline & $\uparrow[69,70]$ & \\
\hline \multirow{2}{*}{ IL-2 } & $\leftrightarrow[63,86]$ & \multirow{2}{*}{$\leftrightarrow$} \\
\hline & $\uparrow[70]$ & \\
\hline \multirow{3}{*}{ IL-4 } & $\leftrightarrow[86,104]$ & \multirow{3}{*}{ NC } \\
\hline & $\uparrow[69,70,77]$ & \\
\hline & $\downarrow[63]$ & \\
\hline \multirow{2}{*}{ IL-6 } & $\leftrightarrow[45,57,77,86,101,102,104]$ & \multirow{2}{*}{$\leftrightarrow$} \\
\hline & $\uparrow[63,70,106]$ & \\
\hline \multirow{3}{*}{ IL-10 } & $\leftrightarrow[48,86]$ & \multirow{3}{*}{ NC } \\
\hline & $\uparrow[44,70]$ & \\
\hline & $\downarrow[104]$ & \\
\hline \multirow{2}{*}{ IL-17 A } & $\leftrightarrow[86]$ & \multirow{2}{*}{ NC } \\
\hline & $\uparrow[70]$ & \\
\hline \multirow{2}{*}{ IL- $1 \beta$} & $\leftrightarrow[60,62,104]$ & \multirow{2}{*}{$\leftrightarrow$} \\
\hline & $\uparrow[45,106]$ & \\
\hline \multirow{2}{*}{ IL-1 ra } & $\leftrightarrow[63,107]$ & \multirow{2}{*}{ NC } \\
\hline & $\uparrow[45,109]$ & \\
\hline IL33 & $\uparrow[58]$ & $\uparrow$ \\
\hline sST2 & $\leftrightarrow[58]$ & $\leftrightarrow$ \\
\hline CCL2 & $\leftrightarrow[45,59,85,104]$ & $\leftrightarrow$ \\
\hline CCL3 & $\leftrightarrow[59,73]$ & $\leftrightarrow$ \\
\hline \multirow{2}{*}{ CXCL8 } & $\leftrightarrow[104]$ & \multirow{2}{*}{ NC } \\
\hline & $\downarrow[59]$ & \\
\hline CXCL10 & $\uparrow[59,86]$ & $\uparrow$ \\
\hline \multirow{2}{*}{ CXCL11 } & $\leftrightarrow[45,85]$ & \multirow{2}{*}{$\mathrm{NC}$} \\
\hline & $\uparrow[59]$ & \\
\hline \multirow{2}{*}{ CXCL24 } & $\uparrow[59]$ & \multirow{2}{*}{ NC } \\
\hline & $\downarrow[85]$ & \\
\hline
\end{tabular}

$\downarrow$ : decreased levels; $\leftrightarrow$ : not altered levels; $\uparrow:$ increased levels; NC: not conclusive.

As mood state seems to influence the expression of cytokines, findings according to different mood states are shown below.

2.3.1. Euthymia. Euthymia has been associated with a mild proinflammatory profile represented mainly by increased sTNFR1 levels (Table 1). Two studies showed that BD patients in euthymia presented increased CXCL10 plasma levels [57, 58]. This finding might suggest a Thl skewed immune response in euthymia as the chemokine CXCL10 is a potent attractant of activated Thl cells. Only one study investigated cytokines in the cerebrospinal fluid of BD patients, reporting increased levels of IL-1 $\beta$ [59]. 
TABLE 2: Cytokine peripheral levels in bipolar disorder patients in mania state compared with controls.

\begin{tabular}{|c|c|c|}
\hline Cytokine & Findings & Conclusion \\
\hline \multirow{2}{*}{ TNF } & $\leftrightarrow[77]$ & \multirow{2}{*}{$\uparrow$} \\
\hline & $\uparrow[101,110,111]$ & \\
\hline \multirow{2}{*}{ sTNFR1 } & $\leftrightarrow[107]$ & \multirow{2}{*}{$\uparrow$} \\
\hline & $\uparrow[61,106]$ & \\
\hline sTNFR2 & $\leftrightarrow[61]$ & $\leftrightarrow$ \\
\hline \multirow{2}{*}{ IL-6 } & $\uparrow[77,111]$ & \multirow{2}{*}{$\leftrightarrow$} \\
\hline & $\leftrightarrow[45,62,64,101,106]$ & \\
\hline IL-4 & $\uparrow[48,77,110]$ & $\uparrow$ \\
\hline \multirow{2}{*}{ IL- $1 \beta$} & $\leftrightarrow[59,110]$ & \multirow{2}{*}{ NC } \\
\hline & $\uparrow[43]$ & \\
\hline IL-12 & $\leftrightarrow[110]$ & $\uparrow$ \\
\hline IL-1 ra & $\leftrightarrow[45,70]$ & $\leftrightarrow$ \\
\hline IL33 & $\uparrow[58]$ & $\uparrow$ \\
\hline sST2 & $\leftrightarrow[58]$ & $\leftrightarrow$ \\
\hline CCL2 & $\leftrightarrow[109]$ & $\leftrightarrow$ \\
\hline CCL3 & $\leftrightarrow[69]$ & $\leftrightarrow$ \\
\hline \multirow[t]{2}{*}{ CXCL8 } & $\uparrow[59]$ & \multirow{2}{*}{ NC } \\
\hline & $\downarrow[69]$ & \\
\hline CXCL10 & $\uparrow[69]$ & $\uparrow$ \\
\hline CXCL11 & $\uparrow[69]$ & $\uparrow$ \\
\hline CXCL24 & $\leftrightarrow[69]$ & $\leftrightarrow$ \\
\hline
\end{tabular}

$\downarrow$ : decreased levels; $\leftrightarrow$ : not altered levels; $\uparrow:$ increased levels; NC: not conclusive.

2.3.2. Mania and Depression. The immune changes already observed in euthymia are enhanced during mania (Table 2) and depressive state (Table 3). BD patients in mania exhibited increased circulating levels of IL-6, TNF- $\alpha$, sTNFR1, IL-ra, and also CXCL10, CXCL11, and IL-4. Besides the association with inflammatory molecules, mania was associated with Th1 (increased CXCL10 [58]) and Th2-type cytokines (increased CXCL11 [60] and IL-4 [48, 59, 61]). BD patients in depression had increased circulating levels of sTNFR1 and CXCL10. It is worth mentioning that the number of studies assessing peripheral cytokines in depressive $\mathrm{BD}$ is much lower than mania or euthymia.

2.3.3. Circulating Cytokine Levels in Bipolar Disorder Compared with Other Psychiatric Disorders. There are few studies comparing $\mathrm{BD}$ with other psychiatric disorders, and it is not possible to draw a definite conclusion on specific immune markers for BD. Regardless the mood state, BD patients did not differ from subjects with schizophrenia on peripheral levels sTNFR1, IL1ra, IL-6, IL-10, and IL-12 [62-65] or from major depression patients when assessing TNF- $\alpha$, IL- 6 , and IL-12 [64, 66-68]. One study found that BD patients during a mood episode (mania or depression) had decreased IL$1 \beta$ levels in comparison with major depression patients [69]. These findings limit the potential use of cytokines and other inflammatory mediators as diagnostic biomarkers for BD.
TABLE 3: Cytokine peripheral levels in bipolar disorder patients in depressive state compared with controls.

\begin{tabular}{lcc}
\hline Cytokine & Findings & Conclusion \\
\hline TNF & $\leftrightarrow[58,59,82,83]$ & $\leftrightarrow$ \\
& $\uparrow[59,66,109]$ & \\
\hline sTNFR1 & $\uparrow[45]$ & $\uparrow$ \\
\hline IL-6 & $\leftrightarrow[45,59,61,66,111]$ & $\leftrightarrow$ \\
\hline IL-4 & $\leftrightarrow[101]$ & $\leftrightarrow[101]$ \\
& $\downarrow[109]$ & NC \\
IL1- $\beta$ & $\leftrightarrow[107,109]$ & $\leftrightarrow$ \\
\hline IL-1 ra & $\uparrow[42]$ & $\leftrightarrow[45]$ \\
\hline & $\uparrow[69]$ & $\leftrightarrow$ \\
\hline der & & \\
\hline
\end{tabular}

$\downarrow$ : decreased levels; $\leftrightarrow$ : not altered levels; $\uparrow:$ increased levels; NC: not conclusive.

2.4. Production of In Vitro Cytokines by Peripheral Mononuclear Blood Cells. In whole blood assays, Kim et al. [70] showed increased levels of IL- 6 and TNF- $\alpha$ following different stimuli (phytohemagglutinin and lipopolysaccharide), but no changes in the production of IL-2, IL-4, and IFN- $\gamma$ in BD patients. Studies evaluating the release of cytokines by stimulated lymphocytes reported lower production of IFN$\gamma[60,65]$ in BD patients compared with controls, with no difference in IL-4 [69] and IL1- $\beta$ [71]. Data regarding the production of IL- $6[67,68]$, IL-2 $[67,68]$, and IL-10 $[60,68$, $72]$ are contradictory, with some studies showing decreased production of IL-2, IL-6, and IL-10 [72], while others report no significant differences $[60,68,71]$.

It seems an apparent paradox the finding of chronic low-grade inflammation in $\mathrm{BD}$, but no response (or even decrease) in cytokines production under immune stimuli in vitro. We hypothesize that the immune system is already overstimulated in $\mathrm{BD}$ patients [73], not being able to respond to additional stimuli.

\section{Cytokines and Their Relationship with Neuroprogression and Comorbidities in Bipolar Disorder Patients}

$\mathrm{BD}$ has been associated with increased peripheral levels of proinflammatory cytokines, and this mild chronic inflammation tends to exacerbate during mood episodes. The frequency of mood episodes (mania or depression) is inversely associated with BD outcome as the higher their frequency, the worst the disease prognosis. Therefore, mood episodes seem to play a pivotal role in $\mathrm{BD}$ progression, and one of the putative mechanisms would be enhanced inflammatory response during mania or bipolar depression [5]. In this scenario, proinflammatory cytokines would act as major "toxic players," contributing to psychopathological changes, cognitive impairment, and related comorbidities and could be regarded as potential biomarkers for neuroprogression in $\mathrm{BD}$ [74-76]. However the studies assessing stage-related 
cytokines changes were cross-sectional, and longitudinal or prospective evidence for this cytokine effect is lacking. For instance, Kauer-Sant'Anna et al. [77] found that the proinflammatory cytokines IL- 6 and TNF- $\alpha$ were elevated in both early- ( $<3$ years of disease) and late-stage ( $>10$ years of disease) $\mathrm{BD}$, whereas the anti-inflammatory cytokine IL10 was increased only in the early stage. Another study described elevated levels of proinflammatory cytokines only in patients with long-duration BD compared with shortduration disease [78]. These preliminary findings suggest that there is a tendency of enhanced inflammation with disease progression.

To date evidence of structural and neuropsychological changes derived, respectively, from neuroimaging and clinical studies supports the concept of staging in BD. Longer lifetime illness duration was related to lower total gray matter, even when controlling for age [7]. Number of manic episodes is associated with cognitive impairment, specifically deficits in executive function, episodic memory, and reduced psychomotor slowing [79].

The neurobiological processes underlying neuroprogression are still undetermined, but inflammatory mechanisms seem to play a major role. TNF- $\alpha$ acts on TNFR1 being capable of inducing neuronal cell death through the activation of caspases and apoptotic machinery $[80,81]$, TNFmediated process might contribute to the volumetric reduction, and hypoactivation of frontal lobes in $\mathrm{BD}[82,83]$ which are associated with disinhibition of limbic structures [84]. This corticolimbic dysfunction may underlie the emotional dysregulation and cognitive impairment associated with $\mathrm{BD}$. In line with this, our and other groups have consistently demonstrated that there is a positive correlation between increased proinflammatory cytokines levels and cognitive impairment, particularly involving frontal lobe functions [ 45 , $85,86]$.

The increased proinflammatory state and the elevated rate of medical comorbidity in $\mathrm{BD}$ patients are also associated [11]. One of the possible pathways is the persistent activation of the HPA axis. Medical conditions, including cardiovascular and endocrine diseases, can cause and are exacerbated by chronic stress and HPA axis activation [83, 84, 87-89]. HPA axis activity presents a major role in the regulation of immune response. Otherwise inflammatory cytokines may stimulate HPA axis activity. Hence mood episodes can trigger stress response-related mechanisms, leading to hyperactivation of the HPA axis and enhanced proinflammatory status contributing to the development and/or exacerbation of the comorbid medical conditions in $\mathrm{BD}$. The medical conditions per se cannot explain the whole proinflammatory state in $\mathrm{BD}$ patients, as differences in relation to controls persist even when controlling for confounding factors. Recently we demonstrated that overweight $\mathrm{BD}$ patients had increased proinflammatory profile when compared with overweight controls, corroborating this assumption [62].

\section{Perspectives}

Current pharmacological therapeutics for $\mathrm{BD}$ are based on neurotransmitters dysfunction. The available strategies are quite effective in relieving behavioral and psychological symptoms mainly related with mania and preventing relapses, but the management of $\mathrm{BD}$ depression remains a great challenge [90]. Moreover, the available drugs do not target and can even worsen medical comorbidities associated with $\mathrm{BD}$ such as obesity and insulin resistance [91].

It is worth mentioning that the mechanisms underlying the therapeutic effect of mood stabilizers and other drugs used in BD are not fully understood. There is preclinical and clinical evidence on these drugs modulating cytokines and playing a role in proinflammatory pathways. Valproic acid decreases the stimulated in vitro proinflammatory cytokines production in healthy controls [92], while lithium, antipsychotics, and antidepressants also inhibit proinflammatory cytokines production and/or synthesis in animal and in vitro studies [93, 94].

The development of new therapeutic targets in the treatment of $\mathrm{BD}$ is highly needed and the immune system is a promising target. In this scenario, anti-inflammatory strategies derived from four major classes, namely, polyunsaturated fatty acids (PUFAs), cyclooxygenase (COX) inhibitors, antiTNF, and minocycline have been tested in BD patients [95]. In a seminal study, Nery et al. [96] conducted a double blind, randomized, placebo-controlled add-on study within depressive or mixed episodes of $\mathrm{BD}$ patients using an $\mathrm{COX}$ 2 inhibitor (celecoxib). In this study the treatment with celecoxib was associated with a faster decrease in depression scores compared with placebo [96]. This study indicates a potential antidepressant effect of anti-inflammatory medications related with the inflammatory/immune imbalance in these patients [96]. New double-blind controlled studies evaluating other anti-inflammatory drugs (i.e., aspirin and minocycline) in $\mathrm{BD}$ patients are underway $[95,97]$. However, a word of caution is necessary as the relation between $\mathrm{BD}$ and inflammation seems to be much more complex than a mere "pro-inflammatory status," represented by elevated circulating levels of cytokines. This can be exemplified by the unexpected clinical observation of a series of cases of mania induced by TNF-antagonists (infliximab and etanercept) [98-100].

\section{Conclusion}

$\mathrm{BD}$ is a complex and multisystemic condition with an underlying neurobiology. Evidences have appointed increased peripheral and central inflammatory cytokines levels in $\mathrm{BD}$ patients. Thus, it is plausible believe that proinflammatory cytokines might contribute to the pathophysiology, clinical comorbidities, and neuroprogression of BD. Future studies should focus on their role as biomarkers of neuroprogression and the evaluation and development of immune-based therapeutic strategies in $\mathrm{BD}$.

\section{Conflict of Interests}

The authors declare that there is no conflict of interests regarding the publication of this paper. 


\section{Acknowledgments}

This work was partly funded by CNPq and Fapemig, Brazil. Dr. Barbosa is recipient of a CAPES postdoctorate scholarship.

\section{References}

[1] K. R. Merikangas, R. Jin, J.-P. He et al., "Prevalence and correlates of bipolar spectrum disorder in the world mental health survey initiative," Archives of General Psychiatry, vol. 68, no. 3, pp. 241-251, 2011.

[2] J. Angst, A. Gamma, R. Sellaro, P. W. Lavori, and H. Zhang, "Recurrence of bipolar disorders and major depression: a lifelong perspective," European Archives of Psychiatry and Clinical Neuroscience, vol. 253, no. 5, pp. 236-240, 2003.

[3] L. L. Judd, H. S. Akiskal, P. J. Schettler et al., "A prospective investigation of the natural history of the long-term weekly symptomatic status of bipolar II disorder," Archives of General Psychiatry, vol. 60, no. 3, pp. 261-269, 2003.

[4] World Health Organization, The Global Burden of Disease: 2004 Update, WHO Press, Geneva, Switzerland, 2008, http://www .who.int/healthinfo/global_burden_disease/GBD_report_ 2004update_full.pdf.

[5] M. Berk, F. Kapczinski, A. C. Andreazza et al., "Pathways underlying neuroprogression in bipolar disorder: focus on inflammation, oxidative stress and neurotrophic factors," Neuroscience and Biobehavioral Reviews, vol. 35, no. 3, pp. 804-817, 2011.

[6] M. R. Schneider, M. P. Delbello, R. K. McNamara, S. M. Strakowski, and C. M. Adler, "Neuroprogression in bipolar disorder," Bipolar Disorders, vol. 14, no. 4, pp. 356-374, 2012.

[7] A. G. Gildengers, K.-H. Chung, S.-H. Huang, A. Begley, H. J. Aizenstein, and S.-Y. Tsai, "Neuroprogressive effects of lifetime illness duration in older adults with bipolar disorder," Bipolar Disorders, vol. 16, no. 6, pp. 617-623, 2014.

[8] M. Berk, "Neuroprogression: pathways to progressive brain changes in bipolar disorder," International Journal of Neuropsychopharmacology, vol. 12, no. 4, pp. 441-445, 2009.

[9] R. J. Schloesser, J. Huang, P. S. Klein, and H. K. Manji, “Cellular plasticity cascades in the pathophysiology and treatment of bipolar disorder," Neuropsychopharmacology, vol. 33, no. 1, pp. 110-133, 2008.

[10] J. Angst, A. Gamma, C. L. Bowden et al., "Evidence-based definitions of bipolar-I and bipolar-II disorders among 5,635 patients with major depressive episodes in the Bridge Study: validity and comorbidity" European Archives of Psychiatry and Clinical Neuroscience, vol. 263, no. 8, pp. 663-673, 2013.

[11] L. Fajutrao, J. Locklear, J. Priaulx, and A. Heyes, "A systematic review of the evidence of the burden of bipolar disorder in Europe," Clinical Practice and Epidemiology in Mental Health, vol. 5, article 3, 2009.

[12] H. A. Swartz and A. Fagiolini, "Cardiovascular disease and bipolar disorder: Risk and clinical implications," Journal of Clinical Psychiatry, vol. 73, no. 12, pp. 1563-1565, 2012.

[13] E. A. Bachen, M. A. Chesney, and L. A. Criswell, "Prevalence of mood and anxiety disorders in women with systemic lupus erythematosus," Arthritis Care \& Research, vol. 61, no. 6, pp. 822-829, 2009.

[14] G. M. Galeazzi, S. Ferrari, G. Giaroli et al., "Psychiatric disorders and depression in multiple sclerosis outpatients: impact of disability and interferon beta therapy," Neurological Sciences, vol. 26, no. 4, pp. 255-262, 2005.

[15] L. J. Edwards and C. S. Constantinescu, "A prospective study of conditions associated with multiple sclerosis in a cohort of 658 consecutive outpatients attending a multiple sclerosis clinic," Multiple Sclerosis, vol. 10, no. 5, pp. 575-581, 2004.

[16] R. W. Kupka, W. A. Nolen, R. M. Post et al., "High rate of autoimmune thyroiditis in bipolar disorder: lack of association with lithium exposure," Biological Psychiatry, vol. 51, no. 4, pp. 305-311, 2002.

[17] W. W. Eaton, M. G. Pedersen, P. R. Nielsen, and P. B. Mortensen, "Autoimmune diseases, bipolar disorder, and non-affective psychosis," Bipolar Disorders, vol. 12, no. 6, pp. 638-646, 2010.

[18] R. Medzhitov, "Origin and physiological roles of inflammation," Nature, vol. 454, no. 7203, pp. 428-435, 2008.

[19] R. Dantzer, J. C. O’Connor, G. G. Freund, R. W. Johnson, and K. W. Kelley, "From inflammation to sickness and depression: when the immune system subjugates the brain," Nature Reviews Neuroscience, vol. 9, no. 1, pp. 46-56, 2008.

[20] S. L. Montgomery and W. J. Bowers, "Tumor necrosis factor- $\alpha$ and the roles it plays in homeostatic and degenerative processes within the central nervous system," Journal of Neuroimmune Pharmacology, vol. 7, no. 1, pp. 42-59, 2012.

[21] S. M. Allan and N. J. Rothwell, "Cytokines and acute neurodegeneration," Nature Reviews Neuroscience, vol. 2, no. 10, pp. 734744, 2001.

[22] R. Dantzer, J. C. O'Connor, M. A. Lawson, and K. W. Kelley, "Inflammation-associated depression: from serotonin to kynurenine," Psychoneuroendocrinology, vol. 36, no. 3, pp. 426436, 2011.

[23] T. W. W. Pace and A. H. Miller, "Cytokines and glucocorticoid receptor signaling: relevance to major depression," Annals of the New York Academy of Sciences, vol. 1179, pp. 86-105, 2009.

[24] P. A. Zunszain, C. Anacker, A. Cattaneo, L. A. Carvalho, and C. M. Pariante, "Glucocorticoids, cytokines and brain abnormalities in depression," Progress in Neuro-Psychopharmacology and Biological Psychiatry, vol. 35, no. 3, pp. 722-729, 2011.

[25] S. P. Gadani, J. C. Cronk, G. T. Norris, and J. Kipnis, "IL-4 in the brain: a cytokine to remember," Journal of Immunology, vol. 189, no. 9, pp. 4213-4219, 2012.

[26] P. A. Zunszain, C. Anacker, A. Cattaneo et al., "Interleukin-1 $\beta$ : a new regulator of the kynurenine pathway affecting human hippocampal neurogenesis," Neuropsychopharmacology, vol. 37, no. 4, pp. 939-949, 2012.

[27] P. McGuffin, F. Rijsdijk, M. Andrew, P. Sham, R. Katz, and A. Cardno, "The heritability of bipolar affective disorder and the genetic relationship to unipolar depression," Archives of General Psychiatry, vol. 60, no. 5, pp. 497-502, 2003.

[28] C.-U. Pae, K.-U. Lee, H. Han, A. Serretti, and T.-Y. Jun, “Tumor necrosis factor alpha gene-G308A polymorphism associated with bipolar I disorder in the Korean population," Psychiatry Research, vol. 125, no. 1, pp. 65-68, 2004.

[29] M. Clerici, B. Arosio, E. Mundo et al., "Cytokine polymorphisms in the pathophysiology of mood disorders," CNS Spectrums, vol. 14, no. 8, pp. 419-425, 2009.

[30] I. V. Meira-Lima, A. C. Pereira, G. F. Mota et al., "Analysis of a polymorphism in the promoter region of the tumor necrosis factor alpha gene in schizophrenia and bipolar disorder: further support for an association with schizophrenia," Molecular Psychiatry, vol. 8, pp. 718-720, 2003. 
[31] F. Middle, I. Jones, E. Robertson, C. Lendon, and N. Craddock, "Tumour necrosis factor $\alpha$ and bipolar affective puerperal psychosis," Psychiatric Genetics, vol. 10, no. 4, pp. 195-198, 2000.

[32] S. Papiol, V. Molina, M. Desco et al., "Gray matter deficits in bipolar disorder are associated with genetic variability at interleukin-1 beta gene (2q13)," Genes, Brain and Behavior, vol. 7, no. 7, pp. 796-801, 2008.

[33] A. Rafiei, S. H. Hosseini, M. Taheri, Z. Hosseni-Khah, M. Hajilooi, and Z. Mazaheri, "Influence of IL-1RN Intron 2 variable number of tandem repeats (VNTR) polymorphism on bipolar disorder," Neuropsychobiology, vol. 67, no. 2, pp. 116-121, 2013.

[34] S. Papiol, A. Rosa, B. Gutiérrez et al., "Interleukin-1 cluster is associated with genetic risk for schizophrenia and bipolar disorder," Journal of Medical Genetics, vol. 41, no. 3, pp. 219-223, 2004.

[35] S. J. Kim, H. J. Lee, H. G. Koo et al., "Impact of IL-1 receptor antagonist gene polymorphism on schizophrenia and bipolar disorder," Psychiatric Genetics, vol. 14, no. 3, pp. 165-167, 2004.

[36] U. T. Hacker, S. Erhardt, K. Tschoèp, T. Jelinek, and S. Endres, "Influence of the IL-1Ra gene polymorphism on in vivo synthesis of IL-1Ra and IL-1 $\beta$ after live yellow fever vaccination," Clinical and Experimental Immunology, vol. 125, no. 3, pp. 465469, 2001

[37] S. Santtila, K. Savinainen, and M. Hurme, "Presence of the IL-1RA allele $2(\operatorname{IL} 1 R N * 2)$ is associated with enhanced IL-1 $\beta$ production in vitro," Scandinavian Journal of Immunology, vol. 47, no. 3, pp. 195-198, 1998.

[38] A. C. Altamura, E. Mundo, E. Cattaneo et al., "The MCP-1 Gene (SCYA2) and mood disorders: preliminary results of a casecontrol association study," NeuroImmunoModulation, vol. 17, no. 2, pp. 126-131, 2010.

[39] M. S. Roh, K. Y. Lee, E. J. Joo, N. Lee, and Y. S. Kim, "No association of the MCP-1 promoter A-2518G polymorphism with bipolar disorder in the Korean population," Neuroscience Letters, vol. 427, no. 1, pp. 1-5, 2007.

[40] C.-U. Pae, J.-J. Kim, H.-S. Yu et al., "Monocyte chemoattractant protein-1 promoter - 2518 polymorphism may have an influence on clinical heterogeneity of bipolar I disorder in the Korean population," Neuropsychobiology, vol. 49, no. 3, pp. 111-114, 2004.

[41] I. Pedroso, A. Lourdusamy, M. Rietschel et al., "Common genetic variants and gene-expression changes associated with bipolar disorder are over-represented in brain signaling pathway genes," Biological Psychiatry, vol. 72, no. 4, pp. 311-317, 2012.

[42] D. T. Chen, X. Jiang, N. Akula et al., "Genome-wide association study meta-analysis of European and Asian-ancestry samples identifies three novel loci associated with bipolar disorder," Molecular Psychiatry, vol. 18, pp. 195-205, 2013.

[43] R. C. Padmos, M. H. J. Hillegers, E. M. Knijff et al., "A discriminating messenger RNA signature for bipolar disorder formed by an aberrant expression of inflammatory genes in monocytes," Archives of General Psychiatry, vol. 65, no. 4, pp. 395-407, 2008.

[44] J.-W. Hsu, J.-F. Lirng, S.-J. Wang et al., "Association of thalamic serotonin transporter and interleukin-10 in bipolar I disorder: a SPECT study," Bipolar Disorders, vol. 16, no. 3, pp. 241-248, 2014.

[45] F. E. Lotrich, M. A. Butters, H. Aizenstein, M. M. Marron, C. F. Reynolds, and A. G. Gildengers, "The relationship between interleukin-1 receptor antagonist and cognitive function in older adults with bipolar disorder," International Journal of Geriatric Psychiatry, vol. 29, no. 6, pp. 635-644, 2014.
[46] J. B. Savitz, J. L. Price, and W. C. Drevets, "Neuropathological and neuromorphometric abnormalities in bipolar disorder: view from the medial prefrontal cortical network," Neuroscience \& Biobehavioral Reviews, vol. 42, pp. 132-147, 2014.

[47] J. S. Rao, G. J. Harry, S. I. Rapoport, and H. W. Kim, "Increased excitotoxicity and neuroinflammatory markers in postmortem frontal cortex from bipolar disorder patients," Molecular Psychiatry, vol. 15, no. 4, pp. 384-392, 2010.

[48] Y. B. Bezchlibnyk, J.-F. Wang, G. M. McQueen, and L. Trevor Young, "Gene expression differences in bipolar disorder revealed by cDNA array analysis of post-mortem frontal cortex," Journal of Neurochemistry, vol. 79, no. 4, pp. 826-834, 2001.

[49] B. Dean, A. S. Gibbons, N. Tawadros, L. Brooks, I. P. Everall, and E. Scarr, "Different changes in cortical tumor necrosis factor$\alpha$-related pathways in schizophrenia and mood disorders," Molecular Psychiatry, vol. 18, no. 7, pp. 767-773, 2013.

[50] J. D. Townsend, S. J. Torrisi, M. D. Lieberman, C. A. Sugar, S. Y. Bookheimer, and L. L. Altshuler, "Frontal-amygdala connectivity alterations during emotion downregulation in bipolar $\mathrm{i}$ disorder," Biological Psychiatry, vol. 73, no. 2, pp. 127-135, 2013.

[51] C. C. Watkins, A. Sawa, and M. G. Pomper, "Glia and immune cell signaling in bipolar disorder: Insights from neuropharmacology and molecular imaging to clinical application," Translational Psychiatry, vol. 4, article e350, 2014.

[52] W. J. Streit, "Microglial senescence: does the brain's immune system have an expiration date?" Trends in Neurosciences, vol. 29, no. 9, pp. 506-510, 2006.

[53] K. Munkholm, J. V. Braüner, L. V. Kessing, and M. Vinberg, "Cytokines in bipolar disorder vs. healthy control subjects: a systematic review and meta-analysis," Journal of Psychiatric Research, vol. 47, no. 9, pp. 1119-1133, 2013.

[54] A. Modabbernia, S. Taslimi, E. Brietzke, and M. Ashrafi, "Cytokine alterations in bipolar disorder: a meta-analysis of 30 studies," Biological Psychiatry, vol. 74, no. 1, pp. 15-25, 2013.

[55] K. Munkholm, M. Vinberg, and L. Vedel Kessing, "Cytokines in bipolar disorder: a systematic review and meta-analysis," Journal of Affective Disorders, vol. 144, no. 1-2, pp. 16-27, 2013.

[56] M. J. Stuart and B. T. Baune, "Chemokines and chemokine receptors in mood disorders, schizophrenia, and cognitive impairment: a systematic review of biomarker studies," Neuroscience \& Biobehavioral Reviews C, vol. 42, pp. 93-115, 2014.

[57] E. Brietzke, M. Kauer-Sant'Anna, A. L. Teixeira, and F. Kapczinski, "Abnormalities in serum chemokine levels in euthymic patients with bipolar disorder," Brain, Behavior, and Immunity, vol. 23, no. 8, pp. 1079-1082, 2009.

[58] I. G. Barbosa, N. P. Rocha, M. E. Bauer et al., "Chemokines in bipolar disorder: trait or state?" European Archives of Psychiatry and Clinical Neuroscience, vol. 263, no. 2, pp. 159-165, 2013.

[59] J. Söderlund, S. K. Olsson, M. Samuelsson et al., "Elevation of cerebrospinal fluid interleukin- $1 \beta$ in bipolar disorder," Journal of Psychiatry and Neuroscience, vol. 36, no. 2, pp. 114-118, 2011.

[60] H.-C. Liu, Y.-Y. Yang, Y.-M. Chou, K.-P. Chen, W. W. Shen, and S.-J. Leu, "Immunologic variables in acute mania of bipolar disorder," Journal of Neuroimmunology, vol. 150, no. 1-2, pp. 116122, 2004.

[61] F. Kapczinski, F. Dal-Pizzol, A. L. Teixeira et al., "Peripheral biomarkers and illness activity in bipolar disorder," Journal of Psychiatric Research, vol. 45, no. 2, pp. 156-161, 2011.

[62] M. Kunz, K. M. Ceresér, P. D. Goi et al., "Serum levels of IL-6, IL-10 and TNF- $\alpha$ in patients with bipolar disorder and schizophrenia: differences in pro- and anti-inflammatory 
balance," Revista Brasileira de Psiquiatria, vol.33, no. 3, pp. 268274, 2011.

[63] S. Hope, I. Dieset, I. Agartz et al., "Affective symptoms are associated with markers of inflammation and immune activation in bipolar disorders but not in schizophrenia," Journal of Psychiatric Research, vol. 45, no. 12, pp. 1608-1616, 2011.

[64] Y.-K. Kim, I.-B. Suh, H. Kim et al., "The plasma levels of interleukin-12 in schizophrenia, major depression, and bipolar mania: effects of psychotropic drugs," Molecular Psychiatry, vol. 7, no. 10, pp. 1107-1114, 2002.

[65] S. Hope, I. Melle, P. Aukrust et al., "Similar immune profile in bipolar disorder and schizophrenia: selective increase in soluble tumor necrosis factor receptor I and von Willebrand factor," Bipolar Disorders, vol. 11, no. 7, pp. 726-734, 2009.

[66] Y.-J. Hung, C.-H. Hsieh, Y.-J. Chen et al., "Insulin sensitivity, proinflammatory markers and adiponectin in young males with different subtypes of depressive disorder," Clinical Endocrinology, vol. 67, no. 5, pp. 784-789, 2007.

[67] K. Becking, L. Boschloo, N. Vogelzangs et al., "The association between immune activation and manic symptoms in patients with a depressive disorder," Translational Psychiatry, vol. 3, article e314, 2013.

[68] S. C. Su, M. T. Sun, M. J. Wen, C. J. Lin, Y. C. Chen, and Y. J. Hung, "Brain-derived neurotrophic factor, adiponectin, and proinflammatory markers in various subtypes of depression in young men," International Journal of Psychiatry in Medicine, vol. 42, no. 3, pp. 211-226, 2011.

[69] R. Mota, M. Gazal, B. A. Acosta et al., "Interleukin- $1 \beta$ is associated with depressive episode in major depression but not in bipolar disorder," Journal of Psychiatric Research, vol. 47, no. 12, pp. 2011-2014, 2013.

[70] Y.-K. Kim, H.-G. Jung, A.-M. Myint, H. Kim, and S.-H. Park, "Imbalance between pro-inflammatory and anti-inflammatory cytokines in bipolar disorder," Journal of Affective Disorders, vol. 104, no. 1-3, pp. 91-95, 2007.

[71] E. M. Knijff, B. M. Nadine, R. W. Kupka et al., "An imbalance in the production of IL- $1 \beta$ and IL- 6 by monocytes of bipolar patients: restoration by lithium treatment," Bipolar Disorders, vol. 9, no. 7, pp. 743-753, 2007.

[72] F. Boufidou, C. Nikolaou, B. Alevizos, I. A. Liappas, and G. N. Christodoulou, "Cytokine production in bipolar affective disorder patients under lithium treatment," Journal of Affective Disorders, vol. 82, no. 2, pp. 309-313, 2004.

[73] I. G. Barbosa, C. R. C. Nogueira, N. P. Rocha et al., "Altered intracellular signaling cascades in peripheral blood mononuclear cells from BD patients," Journal of Psychiatric Research, vol. 47, no. 12, pp. 1949-1954, 2013.

[74] M. H. Rapaportt, "Immune parameters in euthymic bipolar patients and normal volunteers," Journal of Affective Disorders, vol. 32, no. 3, pp. 149-156, 1994.

[75] A. L. Teixeira, I. G. Barbosa, R. MacHado-Vieira, L. B. Rizzo, A. Wieck, and M. E. Bauer, "Novel biomarkers for bipolar disorder," Expert Opinion on Medical Diagnostics, vol. 7, no. 2, pp. 147-159, 2013.

[76] I. G. Barbosa, R. Machado-Vieira, J. C. Soares, and A. L. Teixeira, "The immunology of bipolar disorder," Neuroimmunomodulation, vol. 21, pp. 117-122, 2014.

[77] M. Kauer-Sant'Anna, F. Kapczinski, A. C. Andreazza et al., "Brain-derived neurotrophic factor and inflammatory markers in patients with early- vs. late-stage bipolar disorder," International Journal of Neuropsychopharmacology, vol. 12, no. 4, pp. 447-458, 2009.
[78] I. Grande, P. V. Magalhães, I. Chendo et al., "Staging bipolar disorder: clinical, biochemical, and functional correlates," Acta Psychiatrica Scandinavica, vol. 129, no. 6, pp. 437-444, 2014.

[79] C. López-Jaramillo, J. Lopera-Vásquez, A. Gallo et al., "Effects of recurrence on the cognitive performance of patients with bipolar I disorder: implications for relapse prevention and treatment adherence," Bipolar Disorders, vol. 12, no. 5, pp. 557567, 2010.

[80] E. Cacci, J.-H. Claasen, and Z. Kokaia, "Microglia-derived tumor necrosis factor- $\alpha$ exaggerates death of newborn hippocampal progenitor cells in vitro," Journal of Neuroscience Research, vol. 80, no. 6, pp. 789-797, 2005.

[81] J. A. Smith, A. Das, S. K. Ray, and N. L. Banik, "Role of proinflammatory cytokines released from microglia in neurodegenerative diseases," Brain Research Bulletin, vol. 87, no. 1, pp. 10-20, 2012.

[82] D. A. Kupferschmidt and K. K. Zakzanis, "Toward a functional neuroanatomical signature of bipolar disorder: quantitative evidence from the neuroimaging literature," Psychiatry Research - Neuroimaging, vol. 193, no. 2, pp. 71-79, 2011.

[83] C. Langan and C. McDonald, "Neurobiological trait abnormalities in bipolar disorder," Molecular Psychiatry, vol. 14, no. 9, pp. 833-846, 2009.

[84] J. O. Brooks III, P. W. Wang, J. C. Bonner et al., "Decreased prefrontal, anterior cingulate, insula, and ventral striatal metabolism in medication-free depressed outpatients with bipolar disorder," Journal of Psychiatric Research, vol. 43, no. 3, pp. 181-188, 2009.

[85] I. G. Barbosa, N. P. Rocha, A. S. de Miranda et al., "Increased levels of adipokines in bipolar disorder," Journal of Psychiatric Research, vol. 46, no. 3, pp. 389-393, 2012.

[86] O. Doganavsargil-Baysal, B. Cinemre, U. M. Aksoy et al., "Levels of TNF- $\alpha$, soluble TNF receptors (sTNFR1, sTNFR2), and cognition in bipolar disorder," Human Psychopharmacology, vol. 28, no. 2, pp. 160-167, 2013.

[87] A. H. Miller, V. Maletic, and C. L. Raison, "Inflammation and its discontents: the role of cytokines in the pathophysiology of major depression," Biological Psychiatry, vol. 65, no. 9, pp. 732741, 2009.

[88] R. Havermans, N. A. Nicolson, J. Berkhof, and M. W. deVries, "Patterns of salivary cortisol secretion and responses to daily events in patients with remitted bipolar disorder," Psychoneuroendocrinology, vol. 36, no. 2, pp. 258-265, 2011.

[89] J. Schmider, C.-H. Lammers, U. Gotthardt, M. Dettling, F. Holsboer, and I. J. E. Heuser, "Combined dexamethasone/ corticotropin-releasing hormone test in acute and remitted manic patients, in acute depression, and in normal controls: I," Biological Psychiatry, vol. 38, no. 12, pp. 797-802, 1995.

[90] L. N. Yatham, S. H. Kennedy, S. V. Parikh et al., "Canadian Network for Mood and Anxiety Treatments (CANMAT) and International Society for Bipolar Disorders (ISBD) collaborative update of CANMAT guidelines for the management of patients with bipolar disorder: update 2013," Bipolar Disorders, vol. 15, no. 1, pp. 1-44, 2013.

[91] J. D. Rosenblat, D. S. Cha, R. B. Mansur, and R. S. McIntyre, "Inflamed moods: a review of the interactions between inflammation and mood disorders," Progress in NeuroPsychopharmacology \& Biological Psychiatry, vol. 53, pp. 23-34, 2014.

[92] H. Himmerich, S. Bartsch, H. Hamer et al., "Modulation of cytokine production by drugs with antiepileptic or mood 
stabilizer properties in anti-CD3- and anti-CD40-stimulated blood in vitro," Oxidative Medicine and Cellular Longevity, vol. 2014, Article ID 806162, 11 pages, 2014.

[93] A. Nassar and A. N. Azab, "Effects of lithium on inflammation," ACS Chemical Neuroscience, vol. 5, no. 6, pp. 451-458, 2014.

[94] J. D. Rosenblat, D. S. Cha, R. B. Mansur, and R. S. McIntyre, "Inflamed moods: a review of the interactions between inflammation and mood disorders," Progress in NeuroPsychopharmacology and Biological Psychiatry, vol. 53, pp. 2334, 2014.

[95] G. Fond, N. Hamdani, F. Kapczinski et al., "Effectiveness and tolerance of anti-inflammatory drugs' add-on therapy in major mental disorders: a systematic qualitative review," Acta Psychiatrica Scandinavica, vol. 129, no. 3, pp. 163-179, 2014.

[96] F. G. Nery, E. S. Monkul, J. P. Hatch et al., "Celecoxib as an adjunct in the treatment of depressive or mixed episodes of bipolar disorder: a double-blind, randomized, placebocontrolled study," Human Psychopharmacology, vol. 23, no. 2, pp. 87-94, 2008.

[97] J. Savitz, S. Preskorn, T. K. Teague, D. Drevets, W. Yates, and W. Drevets, "Minocycline and aspirin in the treatment of bipolar depression: a protocol for a proof-of-concept, randomised, doubleblind, placebo-controlled, 232 clinical trial," BMJ Open, vol. 2, no. 1, Article ID 000643, 2012.

[98] K. R. Kaufman, "Etanercept, anticytokines and mania," International Clinical Psychopharmacology, vol. 20, no. 4, pp. 239-241, 2005.

[99] E. Brietzke and B. Lafer, "Induction of manic switch by the tumour necrosis factor- $\alpha$ antagonist infliximab," Psychiatry and Clinical Neurosciences, vol. 64, no. 4, pp. 442-443, 2010.

[100] M. Austin and Y. C. J. Tan, "Mania associated with infliximab," Australian and New Zealand Journal of Psychiatry, vol. 46, no. 7, pp. 684-685, 2012.

[101] E. Brietzke, L. Stertz, B. S. Fernandes et al., "Comparison of cytokine levels in depressed, manic and euthymic patients with bipolar disorder," Journal of Affective Disorders, vol. 116, no. 3, pp. 214-217, 2009.

[102] A. Remlinger-Molenda, P. Wojciak, M. Michalak, J. Karczewski, and J. K. Rybakowski, "Selected cytokine profiles during remission in bipolar patients," Neuropsychobiology, vol. 66, no. 3, pp. 193-198, 2012.

[103] R. C. Drexhage, T. H. Hoogenboezem, M. A. Versnel, A. Berghout, W. A. Nolen, and H. A. Drexhage, "The activation of monocyte and T cell networks in patients with bipolar disorder," Brain, Behavior, and Immunity, vol. 25, no. 6, pp. 1206-1213, 2011.

[104] S. Guloksuz, E. Aktas Cetin, T. Cetin, G. Deniz, E. T. Oral, and D. J. Nutt, "Cytokine levels in euthymic bipolar patients," Journal of Affective Disorders, vol. 126, no. 3, pp. 458-462, 2010.

[105] C. H. Do Prado, L. B. Rizzo, A. Wieck et al., "Reduced regulatory T cells are associated with higher levels of Th1/TH17 cytokines and activated MAPK in type 1 bipolar disorder," Psychoneuroendocrinology, vol. 38, no. 5, pp. 667-676, 2013.

[106] S. Y. Tsai, K. H. Chung, J. Y. Wu, C. J. Kuo, H. C. Lee, and S. H. Huang, "Inflammatory markers and their relationships with leptin and insulin from acute mania to full remission in bipolar disorder," Journal of Affective Disorders, vol. 136, no. 1-2, pp.110116, 2012.

[107] T. Cetin, S. Guloksuz, E. A. Cetin et al., "Plasma concentrations of soluble cytokine receptors in euthymic bipolar patients with and without subsyndromal symptoms," BMC Psychiatry, vol. 12, article 158, 2012.
[108] Y.-K. Kim, A.-M. Myint, B.-H. Lee et al., "T-helper types 1, 2, and 3 cytokine interactions in symptomatic manic patients," Psychiatry Research, vol. 129, no. 3, pp. 267-272, 2004.

[109] I. G. Barbosa, I. B. Morato, A. S. de Miranda, M. E. Bauer, J. C. Soares, and A. L. Teixeira, "A preliminary report of increased plasma levels of IL-33 in bipolar disorder: further evidence of pro-inflammatory status," Journal of Affective Disorders, vol. 157, pp. 41-44, 2014.

[110] S. M. O’Brien, P. Scully, L. V. Scott, and T. G. Dinan, “Cytokine profiles in bipolar affective disorder: focus on acutely ill patients," Journal of Affective Disorders, vol. 90, no. 2-3, pp. 263267, 2006.

[111] M. Maes, E. Bosmans, J. Calabrese, R. Smith, and H. Y. Meltzer, "Interleukin-2 and interleukin-6 in schizophrenia and mania: effects of neuroleptics and mood stabilizers," Journal of Psychiatric Research, vol. 29, no. 2, pp. 141-152, 1995. 

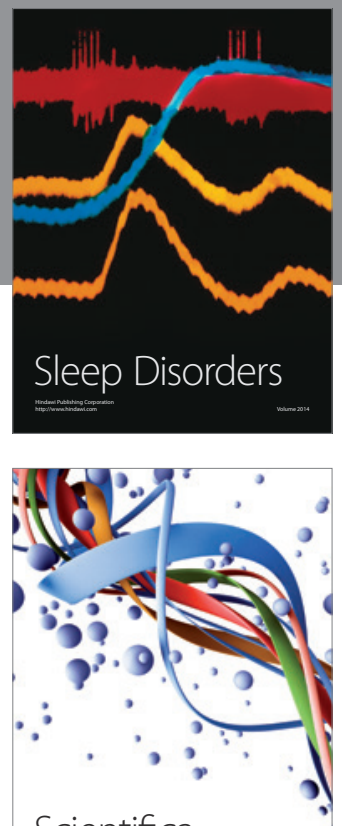

Scientifica
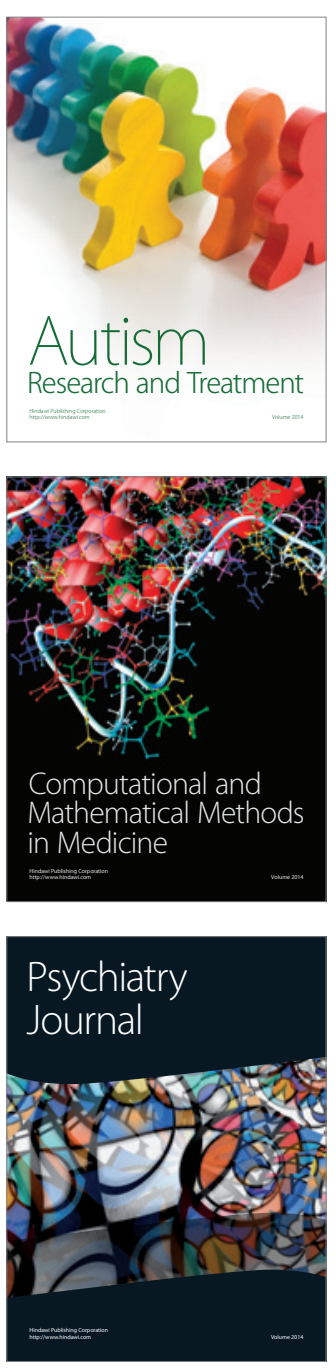
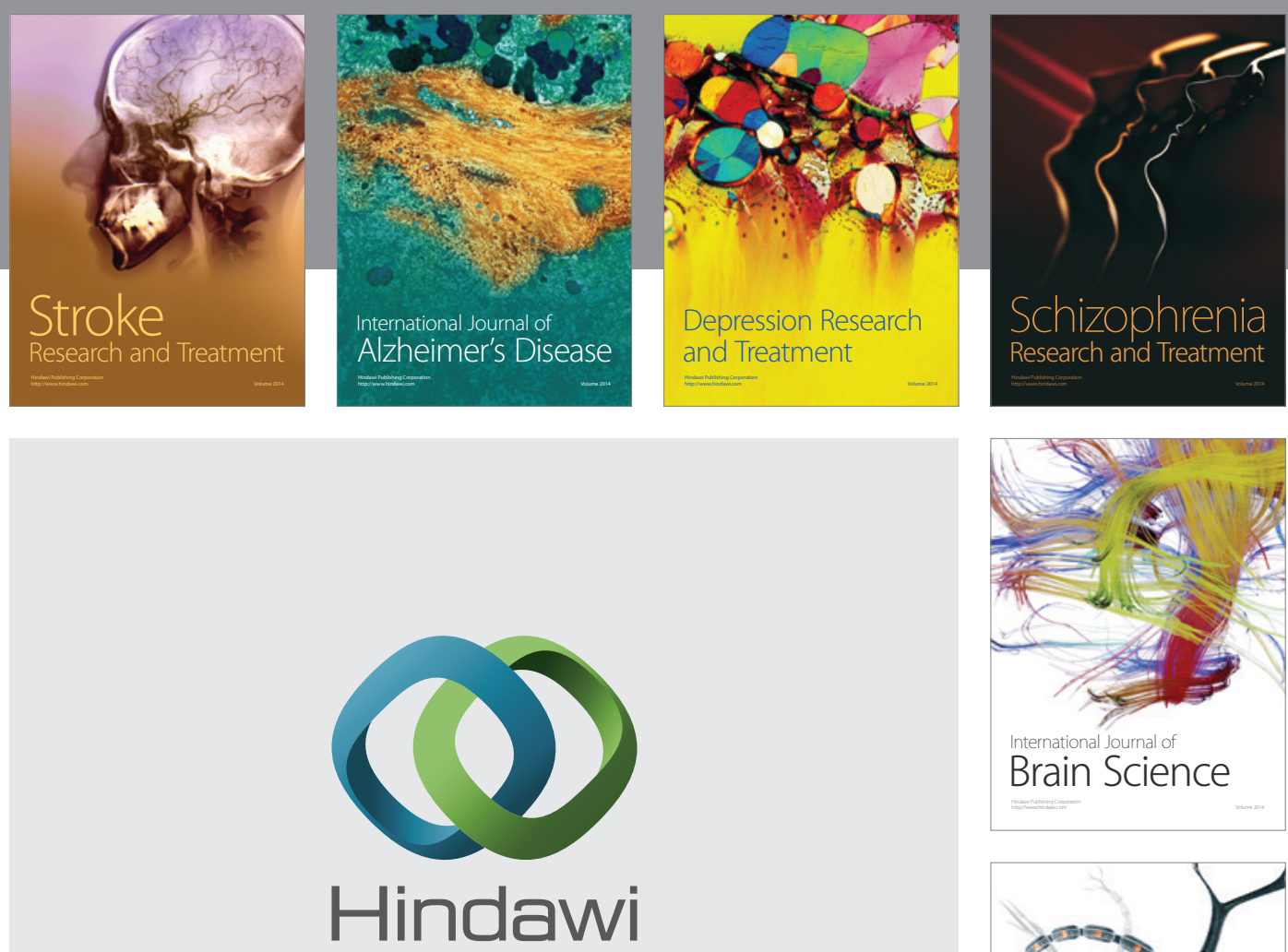

Submit your manuscripts at

http://www.hindawi.com
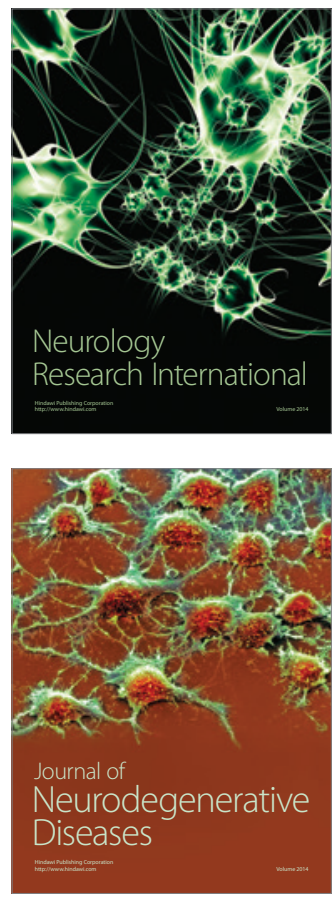

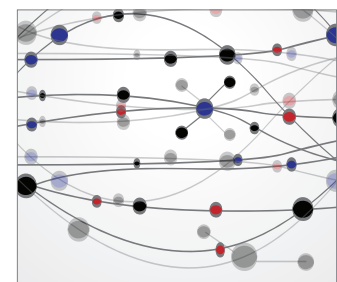

The Scientific World Journal
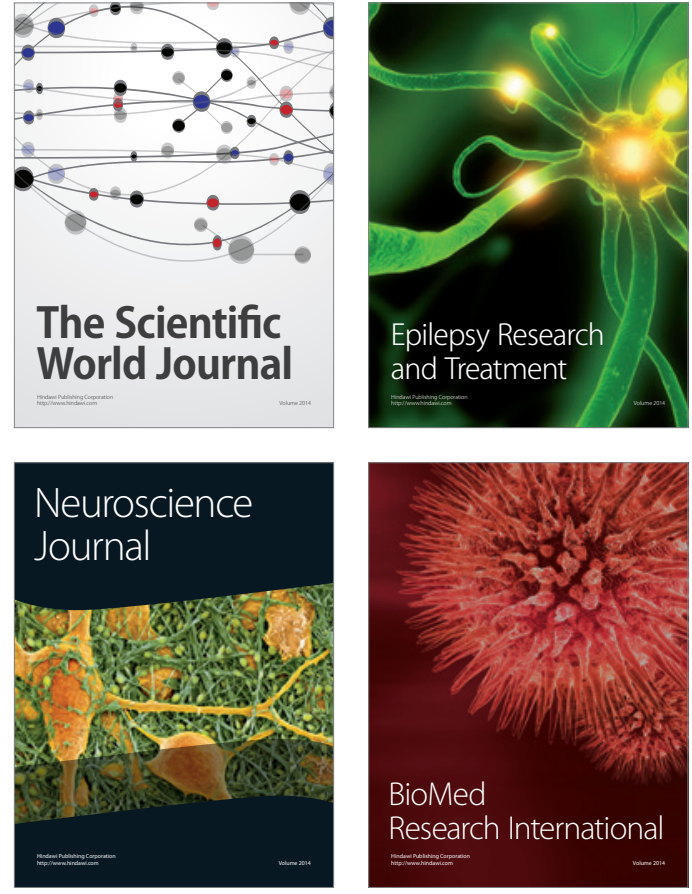

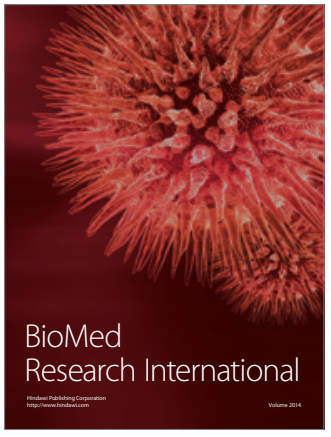

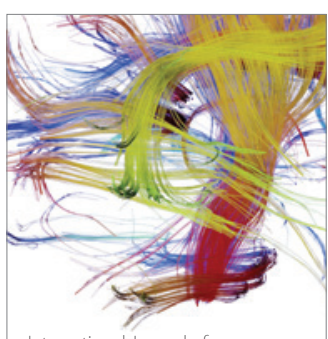

Brain Science

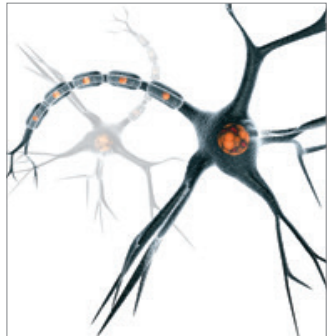

Neural Plasticity
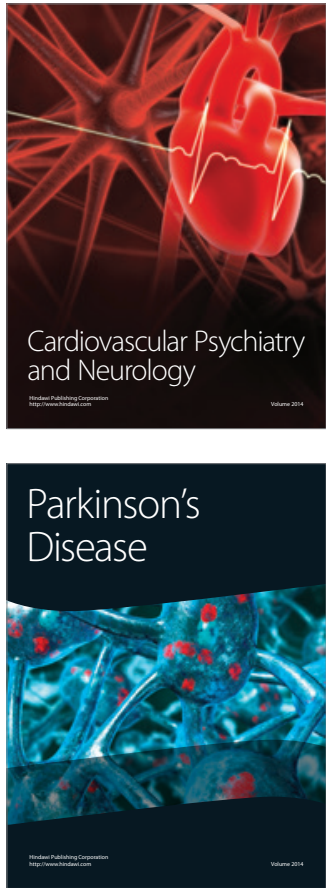